\title{
Hand hygiene compliance and associated factors among health care providers in Central Gondar zone public primary hospitals, Northwest Ethiopia
}

\author{
Garedew Tadege Engdaw, Mulat Gebrehiwot and Zewudu Andualemº
}

\begin{abstract}
Background: Poor hand hygiene compliance is one in all the leading contributory factors to healthcare-associated infections. This is an important source of complications across the continuum of care and poses a serious threat to people admitted to hospitals. However, the magnitude and associated factors of hand hygiene compliance in public primary hospitals were not well investigated in Ethiopia. Therefore, this study was conducted to assess hand hygiene compliance and associated factors among health care providers in Central Gondar Zone public primary hospitals, Northwest Ethiopia.

Methods: An Institutional based cross-sectional study was conducted from March to April 2019 among health care providers. The data were collected using self-administered questionnaires and observational checklists. Using Epi Info 3.1, data was entered and analyzed using SPSS version 23. The data were analyzed using descriptive statistics and logistic regression models. A p-value less than 0.05 with $95 \%$ confidence interval was used to declare statistical significance.

Results: Of 335 study participants, 50 (14.9\%), had good hand hygiene compliance. Training on hand hygiene ( $A O R=8.07,95 \% \mathrm{Cl}: 2.91,22.39$ ), availability of adequate soap and water for hand hygiene ( $\mathrm{AOR}=5.10,95 \% \mathrm{Cl}: 1.93$, 13.52), availability of alcohol-based hand rub ( $A O R=3.23,95 \% \mathrm{Cl}: 1.32,7.92)$, knowledge about hand hygiene ( $\mathrm{AOR}=$ $6.74,95 \% \mathrm{Cl}: 2.96,15.34)$ and attitude towards hand hygiene (AOR $=2.15,95 \% \mathrm{Cl}: 1.04,4.46)$ were factors associated with hand hygiene compliance.

Conclusion: The overall level of hand hygiene compliance among health care providers was poor. Training, availability of adequate soap and water, availability of alcohol-based hand rub, knowledge on hand hygiene, and attitude of health care providers were significantly associated with hand hygiene compliance.
\end{abstract}

Keywords: Hand hygiene compliance, Health care provider, Public primary hospitals

\section{Background}

Hand hygiene is the compliance of cleansing hands with soap and water or with antiseptic hand rub to remove transient microorganisms from hands and maintain the condition of the skin [1]. It is one of the most important health issues in the world and could be a single efficient and sensible life to reduce the incidence of health-

\footnotetext{
* Correspondence: zewuduandualem12@gmail.com
Department of Environmental and Occupational Health and Safety, Institute

* Correspondence: zewuduandualem12@gmail.com
Department of Environmental and Occupational Health and Safety, Institute of Public Health College of Medicine and Health Sciences, University of Gondar, Gondar, Ethiopia
}

(c) The Author(s). 2019 Open Access This article is distributed under the terms of the Creative Commons Attribution 4.0 International License (http://creativecommons.org/licenses/by/4.0/), which permits unrestricted use, distribution, and reproduction in any medium, provided you give appropriate credit to the original author(s) and the source, provide a link to the Creative Commons license, and indicate if changes were made. The Creative Commons Public Domain Dedication waiver (http://creativecommons.org/publicdomain/zero/1.0/) applies to the data made available in this article, unless otherwise stated.

related infections and thereby reveal antimicrobial resistance in all settings, from advanced health care systems to primary health care [2].

Performing hand hygiene activities within the health institutions by health care providers with Alcohol-Based Hand Rub (ABHR) at multiple times causes them to feel uncomfortable. The right technique and duration of handwashing using soap and water and ABHR are very important to confirm the removal of microorganisms. This will be done before and once touching the patient, before handling an invasive device for patient care, once 
contact with body fluids or excretions, mucous membranes, non-intact skin, or wound dressings, moving from a contaminated body web (site) to a different body site throughout care of the identical patient $[3,4]$.

Healthcare-associated infections (HCAIs) have an excellent impact on morbidity, length of hospital stays, and treatment prices [5]. Health care providers' hands are the main usual mode of the vehicle for the transmission of HCAIs. About $50 \%$ of HCAIs happens due to the hand of health care providers [6].

During patient care, unless there are recommended hand hygiene compliance of health care suppliers uninterrupted, hands are contaminated with a microorganism [7]. Annually regarding many numerous patients have suffered from HCAIs worldwide [8]. Improper hand hygiene by HCPs is answerable for regarding $40 \%$ of health facility infections [9]. This infection is answerable for nearly $50 \%$ of the deaths that occur on the far side a pair of weeks of age [3].

Therefore, the aim of this study was to assess hand hygiene compliance and associated factors among health care providers in Central Gondar Zone public primary hospitals, Northwest Ethiopia, 2019.

\section{Methods and materials}

\section{Study design, period, and study area}

The institutional-based cross-sectional study was conducted to assess hand hygiene compliance and associated factors among health care providers in central Gondar zone public primary Hospitals, northwest Ethiopia, from March to April, 2019.

The study was conducted in the region of northwest Amhara in five primary hospitals named, Kolladiba, Aykel, Wogera, Delgi, and Guhala. These hospitals have clinical and administrative staff. The number of health care providers differ from hospital to hospital. Kolladiba (70), Aykel (80), Wogera (66), Delgi (54) and Guhala (71) public primary hospitals.

\section{Eligibility criteria Inclusion criteria}

All health care providers who had worked at least 6 months in the central Gondar zone public primary hospitals who were included in this study.

\section{Exclusion criteria}

Health care providers who were not present during the data collection time due to different reasons were excluded.

\section{Sample size determination}

The required sample was calculated using single population proportion formula with the assumption of the proportion $P$-value is $50 \%$ (there is no previous study), and marginal error 5\%, and a standard Z score of 1.96 corresponding to $95 \%$ confidence interval, by adding $5 \%$ nonresponse rate the final sample size was 403 . But in the study setting, the total number of healthcare providers was 341. Due to a small number and affordable to do research on all health care providers, we have included all HCP from five public primary hospitals.

\section{Data collection tools}

Data were collected by a self-administered questionnaire and observation checklist adapted from different literature $[6,10,11]$. The questionnaire had questions related to socio-demographic, knowledge, attitude, and practice. This questionnaire had both open and close-ended questions. The English version of the questionnaire was translated into the local language (Amharic) and it was translated back into English by the third person to check its consistency. Two days training was given on the data collection tools, questioning techniques, and ethical issues, interview techniques, ways of obtaining the verbal consent and how to interact with respondents as precautions for data collectors and supervisors. The data collectors were Nurses and Environmental health professionals under the supervision of two field supervisors.

\section{Operational definitions \\ Good hand hygiene compliance}

Health care providers who practiced all of the hand hygiene moments from the observational checklist [12].

\section{Poor hand hygiene compliance}

Health care providers who do not practice at least one of the hand hygiene moments from the observational checklist [12].

\section{Data processing and analysis}

For completeness and consistency, the collected data were rechecked. Data were entered in Epi-Info version 3.1 software and exported to SPSS version 23 for further analysis. Descriptive statistics was employed for the socio-demographic characteristics of the respondents. Bivariable and multivariable logistic regressions were carried out to identify significantly associated variables with hand hygiene compliance by backward logistic regression variable selection method. Crude Odds Ratio, and Adjusted Odds Ratio (AOR) with 95\%CI were computed to determine the associated factor of hand hygiene compliance and P-value less 0.05 was considered as declared statistically significant. Hosmer and Lemeshow goodness of fit $(P>0.05)$ were used to test the fitness of the model during analysis. 


\section{Results}

Socio-demographic characteristics of respondents

A total of 341 respondents were aimed for this study. Of these, 335 respondents were recorded with a response rate of $98.2 \%$. The mean age of the respondents was $28 \pm 5.6$ ( \pm SD) years. Most of the 299 (89.3\%) study respondents were Orthodox Christians in religion. Professional respondents were 120 (35.8\%) Nurses (Table 1).

\section{Reasons for not practicing hand hygiene}

Half of the study participants argue that the inaccessibility of sink and ABHR is a reason for not practicing good hand hygiene (Fig. 1).

Table 1 Socio-demographic characteristics of the health care providers Central Gondar Zone public primary hospitals, Northwest Ethiopia, 2019 ( $n=335)$

\begin{tabular}{|c|c|c|c|}
\hline Variable & & Frequency & Percent (\%) \\
\hline \multirow[t]{3}{*}{ Age } & $18-24$ & 55 & 16.4 \\
\hline & $25-34$ & 243 & 72.5 \\
\hline & $>35$ & 37 & 11.1 \\
\hline \multirow[t]{2}{*}{ Sex } & Male & 238 & 71 \\
\hline & Female & 97 & \\
\hline \multirow[t]{2}{*}{ Religion } & Orthodox & 299 & 89.3 \\
\hline & Muslima & 36 & 10.7 \\
\hline \multirow[t]{5}{*}{ Profession } & Physician & 41 & 12.2 \\
\hline & Nurse & 120 & 35.8 \\
\hline & Laboratory & 34 & 10.1 \\
\hline & Midwives & 74 & 22.1 \\
\hline & Others $^{b}$ & 66 & 19.7 \\
\hline \multirow[t]{3}{*}{ Level of education } & Diploma & 87 & 26 \\
\hline & Bachelor & 200 & 59.7 \\
\hline & 2nd degree and above ${ }^{c}$ & 48 & 14.3 \\
\hline \multirow[t]{2}{*}{ Marital status } & Married & 159 & 47.5 \\
\hline & Single ${ }^{d}$ & 176 & 52.5 \\
\hline \multirow[t]{4}{*}{ Working experience } & $0-5$ & 201 & 60 \\
\hline & $6-10$ & 63 & 18.8 \\
\hline & $11-15$ & 36 & 10.7 \\
\hline & $\geq 16$ & 35 & 10.5 \\
\hline \multirow[t]{7}{*}{ Unit of work } & OPD & 74 & 22.1 \\
\hline & Emergency & 36 & 10.7 \\
\hline & Inpatient & 53 & 15.8 \\
\hline & Laboratory & 34 & 10.1 \\
\hline & OR & 41 & 12.2 \\
\hline & GYN Obs & 63 & 18.8 \\
\hline & Others ${ }^{\mathrm{e}}$ & 34 & 10.1 \\
\hline
\end{tabular}

${ }^{\text {a }}$ Protestant, Others

${ }^{\mathrm{b}} \mathrm{HO}$, Radiography, Anaesthesia, Optometry, pharmacy

'Masters and GP

${ }^{\mathrm{d}}$ Divorced, Widowed,

'Triage
Hand hygiene compliance of health care providers

In this study, the prevalence of hand hygiene compliance from observation was 14.9\%, (95\% CI: 11.3, 18.5). Above half $181(54 \%)$ of the respondents were knowledgeable about hand hygiene compliance. Of the respondents, 140 $(41.8 \%)$ health care providers are trained about hand hygiene compliance and 177 (52.8\%) assured the presence of alcohol-based hand rub in their working area (Table 2).

\section{Factors associated with hand hygiene compliance}

In bivariable logistic regression; knowledge of six steps of hand hygiene, knowledge on five moments of hand hygiene, having training for hand hygiene, presence of promotion for hand hygiene in the hospital, having adequate soap and water for hand hygiene, presence of adequate individual/wall matted alcohol-based hand rub for hand hygiene, presence of posters for hand hygiene, presence of protocol for hand hygiene, knowledge on hand hygiene compliance and attitude were found to be significantly associated variables with the hand hygiene compliance of health care providers.

After fitting these variables in multivariable logistic regressions; having trained for hand hygiene, have adequate soap \& water for hand hygiene, presence of an adequate individual or wall matted alcohol-based hand rub, knowledge on hand hygiene compliance, and the attitude were significantly associated with the hand hygiene compliance. Study participants who have taken training about hand hygiene were 8.07 times more likely to have good hand hygiene compliance than those who have not taken training ( $\mathrm{AOR}=8.07,95 \% \mathrm{CI}: 2.91,22.39)$. Study participants who have adequate soap and water for handwashing were 5.10 times more likely to have good hand hygiene compliance as compared to study participants who have not adequate soap and water for handwashing ( $A O R=5.10$, 95\%CI: 1.93, 13.52). The odds of having good hand hygiene compliance were 3.23 times more likely who have alcohol-based hand rub individually/wall matted than those who did not have alcohol-based hand rub individually/wall matted. $(\mathrm{AOR}=3.23,95 \% \mathrm{CI}$ : 1.32, 7.92). Those who were knowledgeable of hand hygiene were 6.74 times more likely to have good handwashing compliance than those who were not knowledgeable for hand hygiene (AOR $=6.74,95 \% C I: 2.96,15.34)$. Furthermore, Study participants who had a positive attitude towards hand hygiene were 2.15 times more likely to have good hand hygiene compliance as compared to who have a negative attitude for hand hygiene $(\mathrm{AOR}=2.15,95 \%$ CI: $1.04,4.46$ ) (Table 3).

\section{Discussion}

Poor hand hygiene compliance of health care providers and its healthcare-associated infections have a greater impact on the patients in health care settings [13]. 


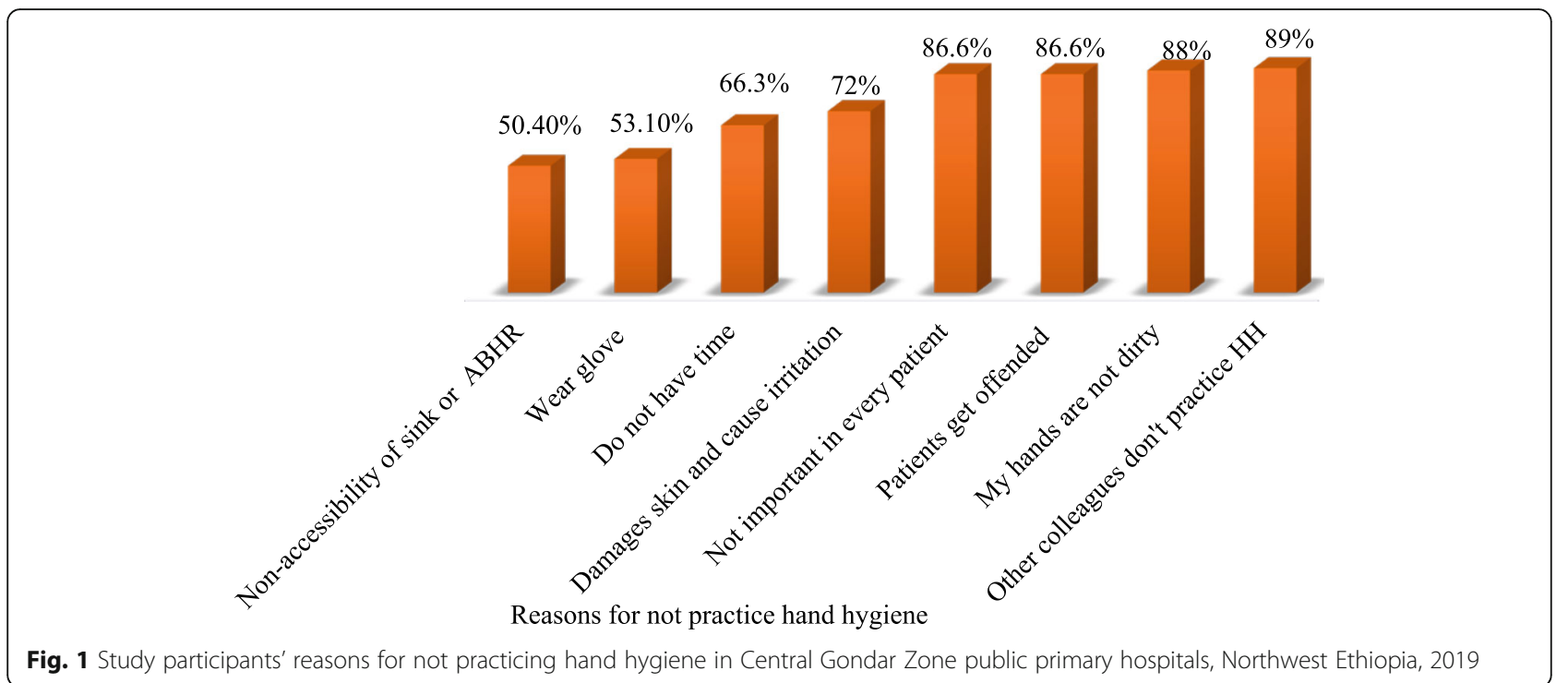

The present study demonstrated that overall good hand hygiene compliance was $14.9 \%$. The finding the study was in line with other study conducted in University of Gondar teaching hospital, Ethiopia 16.5\% [11] and lower than a studies conducted in Mali 21.8\% [14],
Kuwait 33.4\% [15], India 43.4\% [16] and black lion hospital, Ethiopia 79\% [10]. But, this finding was higher as compared with a study carried out in Wachemo University teaching Hospital, Ethiopia 9.2\% [17]. The variation might be due to study setting, sample size, a lack of

Table 2 Variables related to hand hygiene compliance of health care providers in Central Gondar Zone public primary hospitals, Northwest Ethiopia, 2019 ( $n=335)$

\begin{tabular}{|c|c|c|c|}
\hline Variables & & Frequency & Percent (\%) \\
\hline \multirow[t]{2}{*}{ Knowledge } & Good & 181 & 54 \\
\hline & Poor & 154 & 46 \\
\hline \multirow[t]{2}{*}{ Frequently keep hand hygiene } & Yes & 301 & 89.9 \\
\hline & No & 34 & 10.1 \\
\hline \multirow[t]{2}{*}{ Taking training } & Yes & 140 & 41.8 \\
\hline & No & 195 & 58.2 \\
\hline \multirow[t]{2}{*}{ Hospital promotes the importance of $\mathrm{HH}$ to the staffs } & Yes & 161 & 48.1 \\
\hline & No & 174 & 51.9 \\
\hline \multirow[t]{2}{*}{ The presence of soap and water } & Yes & 290 & 86.6 \\
\hline & No & 45 & 13.4 \\
\hline \multirow[t]{2}{*}{ The presence of hand washing sink } & Yes & 162 & 48.4 \\
\hline & No & 173 & 51.6 \\
\hline \multirow[t]{2}{*}{ The presence of wall mount/ individual ABHR } & Yes & 177 & 52.8 \\
\hline & No & 158 & 47.2 \\
\hline \multirow[t]{2}{*}{ The presence of gloves } & Yes & 266 & 79.4 \\
\hline & No & 69 & 20.6 \\
\hline \multirow[t]{2}{*}{ The presence of posters } & Yes & 137 & 40.9 \\
\hline & No & 198 & 59.1 \\
\hline \multirow[t]{2}{*}{ The presence of a protocol level } & Yes & 106 & 31.6 \\
\hline & No & 229 & 68.4 \\
\hline \multirow[t]{2}{*}{ Attitude } & Positive & 183 & 54,6 \\
\hline & Negative & 152 & 45.4 \\
\hline
\end{tabular}


Table 3 Bivariable and multivariable logistic regression analysis of factors associated with hand hygiene compliance among health care providers in Central Gondar Zone public primary hospitals, Northwest Ethiopia, 2019 ( $n=335)$

\begin{tabular}{|c|c|c|c|c|c|}
\hline \multirow[t]{2}{*}{ Variables } & \multicolumn{3}{|c|}{ Hand hygiene compliance } & \multirow[t]{2}{*}{ COR $(95 \% \mathrm{Cl})$} & \multirow[t]{2}{*}{ AOR $(95 \% \mathrm{Cl})$} \\
\hline & & Good & Poor & & \\
\hline \multirow[t]{2}{*}{ Knowing six steps of hand hygiene } & Yes & 43 & 202 & $2.52(1.09,5.84)$ & $1.36(.47,3.91)$ \\
\hline & No & 7 & 83 & 1 & 1 \\
\hline \multirow[t]{2}{*}{ Knowing five movements of hand hygiene } & Yes & 45 & 229 & $5.87(1.38,24.88)$ & $3.5(.16,5.65)$ \\
\hline & No & 5 & 56 & 1 & 1 \\
\hline \multirow[t]{2}{*}{ Trained for hand hygiene } & Yes & 45 & 165 & $6.55(2.52,16.98)$ & $8.07(2.91,22.39)^{\mathrm{c}}$ \\
\hline & No & 5 & 120 & 1 & 1 \\
\hline \multirow[t]{2}{*}{ Promotion for $\mathrm{HH}$ in Hospital } & Yes & 32 & 129 & $2.15(1.15,4.01)$ & $1.57(.74,3.33)$ \\
\hline & No & 18 & 156 & 1 & 1 \\
\hline \multirow[t]{2}{*}{ Have adequate soap \& water for hand hygiene } & Yes & 44 & 190 & $3.67(1.51,8.91)$ & $5.10(1.93,13.52)^{a}$ \\
\hline & No & 6 & 95 & 1 & 1 \\
\hline \multirow[t]{2}{*}{ Presence of alcohol hand rub for individual } & Yes & 43 & 156 & $5.07(2.21,11.68)$ & $3.23(1.32,7.92)^{a}$ \\
\hline & No & 7 & 129 & 1 & 1 \\
\hline \multirow[t]{2}{*}{ Presence of posters for hand hygiene } & Yes & 28 & 109 & $2.06(1.12,3.77)$ & $.99(.36,2.67)$ \\
\hline & No & 22 & 176 & 1 & 1 \\
\hline \multirow[t]{2}{*}{ Presence of protocol level for hand hygiene } & Yes & 26 & 80 & $2.78(1.51,5.12)$ & $1.31(.60,2.85)$ \\
\hline & No & 24 & 205 & 1 & 1 \\
\hline \multirow[t]{2}{*}{ Knowledge about hand hygiene } & Good & 41 & 140 & $4.71(2.21,10.07)$ & $6.74(2.96,15.34)^{a}$ \\
\hline & Poor & 9 & 145 & 1 & 1 \\
\hline \multirow[t]{2}{*}{ Attitude towards hand hygiene } & Positive & 34 & 149 & $1.94(1.03,3.67)$ & $2.15(1.04,4.46)^{a}$ \\
\hline & Negative & 16 & 136 & 1 & 1 \\
\hline
\end{tabular}

${ }^{\mathrm{a} S t a t i s t i c a l l y ~ s i g n i f i c a n t ~ a t ~} a=0.05,1=$ Reference group

awareness on healthcare-associated infections among health care providers, passive Infection Prevention and Control Committees (IPCCs) holding nonsystematic hand hygiene training and audits, inaccessibility of hand hygiene resources and it might be due availability of hand hygiene products and facilities. Alcoholic disinfectants were only used for disinfection of patients' skin prior to aseptic procedures [18].

Knowledge of hand hygiene was associated with hand hygiene compliance. As a result, those who have good knowledge of $\mathrm{HH}$ had 6.74 times more compliance than those who have poor knowledge. This was in line with other similar study done in Kuwait which showed that knowledge of HCPs was significantly associated with good $\mathrm{HH}$ compliance [15]. The possible explanation might be due to knowledge on hand hygiene compliance will help to comply with hand hygiene with recommended way, knowledge will help to identify the advantage and disadvantages of hand hygiene compliance and identify the way of HCAIs transmission and how it is prevented.

Trained health care providers for hand hygiene were 8.07 times more likely to have good hand hygiene compliance than those who were not trained health care providers. Other studies were done in India [19], United
Kingdom [20] and China [21] also showed that training had a positive relationship with $\mathrm{HH}$ compliance in all medical staff. This may be due to the fact that training built the knowledge of health care providers which had a significant association in $\mathrm{HH}$ compliance and those HCPs who had got training is expected to be a role model for others in terms of practicing good $\mathrm{HH}$, Knowledge of HCPs will help to identify risk and benefits practice on the way of HCAIs transmission and how to prevent. A single lecture on basic hand hygiene protocols had a significant and sustained effect in enhancing hand hygiene compliance in a Swedish hospital [22]. A study conducted in University hospital in central Ethiopia showed compliance with hand hygiene at baseline and at follow up after training have a significant relationship with compliance [18].

The attitude was found to be significantly associated with $\mathrm{HH}$ compliance. As a result, those who had a positive attitude on hand hygiene had 2 times more compliance than a negative attitude. This was in line with other similar study done in Jordan which showed that the attitude of health care providers was significantly associated with good hand hygiene compliance [23]. Different reasons can be suggested for this, including the light 
workload they might have, the presence of Water Sanitation and Hygiene (WASH) committee, the presence of positive peer pressure, good professional attitude towards hand hygiene compliance, social factors, direct instruction from the respected body, personal experience, media and educational and religious institutions.

The odds of having good hand hygiene compliance were 3.23 times more likely who have alcohol-based hand rub individually/wall matted than those who did not get alcohol-based hand rub individually/wall matted. This is in line with other studies done in Taiwan [24] and Brazil [25]. The availability of alcohol-based hand rub resulted in significant improvement in hand hygiene compliance of health care providers. This might be due to the presence of alcohol-based hand rub the best way of improving hand hygiene compliance, the presence of alcohol-based hand rub at point of care was a reminder to health care providers to do hand hygiene and it might be easy for implementing hand hygiene. Inaccessibility of resources in their nearby ward might be one of the reasons for not practicing hand hygiene.

The other possible reasons are product selection on the antimicrobial profile, user acceptance, and cost. Additional activity against fungi (including molds), mycobacteria, and bacterial spores may be relevant in high-risk wards or during outbreaks [2] and they are often available as a gel, or on wipes. a study supports the fact that interactive educational programs combined with free availability of hand disinfectants significantly increased hand hygiene compliance [22]. Therefore, it is an excellent alternative to hand hygiene when antimicrobial efficacy, time for the procedure, and limited access to sinks are of concern [26].

The use of WHO advocated alcohol-based hand rubs is a practical solution to overcome these constraints because these can be distributed individually to staff for pocket carriage and placed at the point of care. The major advantage is that its use is well applicable to situations typical of developing countries, such as two patients sharing the same bed, or patient's relatives being requested to help in care provision [14].

Health care providers who got adequate soap and water for handwashing were 5.10 times more likely to had good hand hygiene compliance as compared to health professionals who had not got adequate soap and water for handwashing. This is in line with the study done in Black Lion hospital, Ethiopia [10] but, there was no significant relation with adequate soap and water and hand hygiene compliance from a study conducted in Gondar university teaching hospital, Ethiopia [11]. This may be due to the difference in hospitals setting, availability, and accessibility of resources, user acceptance, and cost.

Like in other developing countries, the priority given to prevention and control of healthcare-associated infection is minimal. This is primarily due to lack of infrastructure, trained manpower, surveillance systems, poor sanitation, overcrowding and understaffing of hospitals, the unfavorable social background of population, lack of legislation mandating accreditation of hospitals and a general attitude of non-compliance amongst health care providers towards even basic procedures of infection control [8].

Improvement strategies of poor hand hygiene compliance among health care providers by ensuring that the necessary infrastructure and products are in place to allow hand hygiene performance at the point of care. This includes access to a safe, continuous water supply and the availability of soap and disposable towels, availability of effective and well-tolerated alcohol-based hand rub products at the point of care [2, 3, 27, 28].

Provision of regular training to all healthcare workers is essential to heighten awareness of microbial transmission by hands, emphasize the importance of hand hygiene and its indications, and to demonstrate the correct procedures for hand rubbing and hand washing. It may be achieved using regular presentations, e-learning modules, posters, focus groups, reflective discussion, videos, self-learning modules, practical demonstrations, feedback from assessment, or combinations of these and other methods and hand hygiene compliance health care providers may improve through placing reminders and prompts (posters, stickers, voice prompts, leaflets, gadgets, etc.) related to the importance of hand hygiene and the appropriate indications and procedures for its performance [29-36].

As far as a self-reported self-administered questionnaire the study may prone to social desirability bias. Since the study was done in public primary hospitals it may lack generalizability for private primary hospital.

\section{Conclusions}

Hand hygiene compliance among health care providers in Central Gondar Zone public primary hospital was poor as compared to the WHO threshold. Training, attitude, the presence of alcohol-based hand rub in the working area, the presence of adequate soap and water in the working area were significantly associated with hand hygiene compliance. Implementing five movements of hand hygiene are the best method for preventing healthcare-associated infections. Therefore, the health care provider should be followed this principle to fight healthcare-associated infections.

\footnotetext{
Abbreviations

AOR: Adjusted odds ratio; COR: Crude odds ratio; HCAl: Healthcare-associated Infections; HCP: Health care providers; HH: Hand hygiene; WHO: World Health Organization

\section{Acknowledgments}

We are highly indebted to the College of Medicine and Health Sciences, University of Gondar, for supporting this research project. We would like to extend our thanks to Amhara regional state, all hospital administration for permission to conduct the study. We would also like to extend our appreciation to the study participants, supervisors and data collectors.
} 


\section{Authors' contributions}

All stated authors GTE, MG, ZA are involved in the study from the inception to design, acquisition of data, analysis and interpretation and drafting of the manuscript. All authors read and approved the final manuscript.

\section{Funding}

No funding source.

\section{Availability of data and materials}

Data will be made available upon the reasonable request to the primary author.

\section{Ethics approval and consent to participate}

Ethical clearance was approved and obtained from the Institutional Review Board of the University of Gondar College of Medicine and Health Sciences, Institute of Public Health. Then the official permission letter was collected from the Central Gondar Zone health department and from each Public Primary Hospital admin offices. Written informed consent was obtained from each study participant after they were included in this study. The purpose of the study was explained to study participants before giving consent. We deliver information on the right to interrupt the observation and refuse to fill the self-administered questionnaires.

\section{Consent for publication}

Not applicable.

\section{Competing interests}

The authors declare that they have no competing interests.

Received: 28 August 2019 Accepted: 24 October 2019

Published online: 26 November 2019

\section{References}

1. Pittet D. Improving adherence to hand hygiene practice: a multidisciplinary approach. Emerg Infect Dis. 2001;7(2):234.

2. Mathur P. Hand hygiene: back to the basics of infection control. Indian J Med Res. 2011;134(5):611.

3. Boyce JM, Pittet D. Guideline for hand hygiene in health-care settings: recommendations of the healthcare infection control practices advisory committee and the HICPAC/SHEA/APIC/IDSA hand hygiene task force. Infect Control Hosp Epidemiol. 2002;23(S12):S3-S40.

4. Pittet D, Allegranzi B, Boyce J. Experts WHOWAfPSFGPSCCGo. The World Health Organization guidelines on hand hygiene in health care and their consensus recommendations. Infect Control Hosp Epidemiol. 2009;30(7):611-22.

5. Pittet D, Allegranzi B, Sax H, Bertinato L, Concia E, Cookson B, et al. Considerations for a WHO European strategy on health-care-associated infection, surveillance, and control. Lancet Infect Dis. 2005;5(4):242-50.

6. Martín-Madrazo C, Salinero-Fort MA, Abanades-Herranz JC, Arnal-Selfa R, García-Ferradal I, Espejo-Matorral F, et al. Effectiveness of a training programme to improve hand hygiene compliance in primary healthcare. BMC Public Health. 2009;9(1):469.

7. Allegranzi B, Storr J, Dziekan G, Leotsakos A, Donaldson L, Pittet D. The first global patient safety challenge "clean care is safer care": from launch to current progress and achievements1. J Hosp Infect. 2007;65:115-23.

8. Mathai E, Allegranzi B, Kilpatrick C, Pittet D. Prevention and control of health care-associated infections through improved hand hygiene. Indian J Med Microbiol. 2010;28(2):100

9. Chang B, Frendl G. Nosocomial infections; 2015. p. 494-8

10. Negewo A, Tsige $Y$, Abebe F. Assessment of hand hygiene practice and factor affecting compliance among nurses in black lion specialized referral hospital. Addis Ababa, Ethiopia; 2017.

11. Abdella N, Alemayehu M, Eredie A, Landers T, Malefia Y, Alene K. Hand hygiene compliance and associated factors among health care providers in Gondar University hospital, Gondar, north West Ethiopia. BMC Public Health. 2014;14:96.

12. World Health Organization. Hand hygiene self-assessment framework WHO patient safety clean your hand save lives, introduction and user instructions; 2010.

13. Erasmus V, Brouwer W, Van Beeck E, Oenema A, Daha T, Richardus JH, et al. A qualitative exploration of reasons for poor hand hygiene among hospital workers lack of positive role models and of convincing evidence that hand hygiene prevents cross-infection. Infect Control Hosp Epidemiol. 2009;30(5): 415-9.
14. Allegranzi B, Sax H, Bengaly L, Riebet H, Minta DK, Chraiti M-N, et al Successful implementation of the World Health Organization hand hygiene improvement strategy in a referral hospital in Mali, Africa. Infect Control Hosp Epidemiol. 2010;31(2):133-41.

15. Al-Wazzan B, Salmeen Y, Al-Amiri E. Abul aa, Bouhaimed M, Al-Taiar a. hand hygiene practices among nursing staff in public secondary care hospitals in Kuwait: self-report and direct observation. Med Princ Pract. 2011;20(4):326-31.

16. Sharma S, Sharma S, Puri S, Whig J. Hand hygiene compliance in the intensive care units of a tertiary care hospital. Indian J Community Med. 2011;36(3):217.

17. Ayele $Y$, Addise A. Hand hygiene compliance and associated factors among health professionals in Wachemo University hospital, Hossaena, south West Ethiopia. IJIRD. 2017;6:28-35.

18. Pfäfflin F, Tufa T, Mesfun MG, Nigussie T, Schönfeld A, Häussinger D, et al. Implementation of the WHO multimodal hand hygiene improvement strategy in a University Hospital in Central Ethiopia. Antimicrob Resist Infect Control. 2017;6:3.

19. Suchitra J, Devi NL. Impact of education on knowledge, attitudes and practices among various categories of health care workers on nosocomial infections. Indian J Med Microbiol. 2007;25(3):181.

20. Randle J, Clarke M, Storr J. Hand hygiene compliance in healthcare workers. J Hosp Infect. 2006;64(3):205-9.

21. Lam BC, Lee J, Lau Y. Hand hygiene practices in a neonatal intensive care unit: a multimodal intervention and impact on nosocomial infection. Pediatrics. 2004;114(5):e565-e71.

22. Sjöberg $M$, Eriksson M. Hand disinfectant practice: the impact of an education intervention. Open Nurs J. 2010;4:20.

23. Al-Hussami M, Darawad M, Almhairat II. Predictors of compliance handwashing practice among healthcare professionals. Healthc Inf. 2011;16(2):79-84.

24. Chen Y-C, Sheng W-H, Wang J-T, Chang S-C, Lin H-C, Tien K-L, et al. Effectiveness and limitations of hand hygiene promotion on decreasing healthcare-associated infections. PLoS One. 2011;6(11):e27163.

25. Santana SL, Furtado GH, Coutinho AP, Medeiros EA. Assessment of healthcare professionals' adherence to hand hygiene after alcohol-based hand rub introduction at an intensive care unit in Sao Paulo, Brazil. Infect Control Hosp Epidemiol. 2007;28(3):365-7.

26. Widmer AF. Replace hand washing with use of a waterless alcohol hand rub? Clin Infect Dis. 2000;31(1):136-43.

27. Rosenberg A. Hand hygiene barriers faced byHealth Care Workers in the Gambia: a health belief model approach; 2016.

28. Rynga D, Kumar S, Gaind R, Rai AK. Hand hygiene compliance and associated factors among health care workers in a tertiary care hospital: self-reported behavior and direct observation. Int J Infect Control. 2017; 13(1):689.

29. Al Kadi A, Salati SA. Hand hygiene practices among medical students. Interdiscip Perspect Infect Dis. 2012;2012:1.

30. Albright J, White B, Pedersen D, Carlson P, Yost L, Littau C. Use patterns and frequency of hand hygiene in healthcare facilities: analysis of electronic surveillance data. Am J Infect Control. 2018;46(10):1104-9.

31. Alp E, Ozturk A, Guven M, Celik I, Doganay M, Voss A. Importance of structured training programs and good role models in hand hygiene in developing countries. J Infect Public health. 2011;4(2):80-90.

32. Chavali S, Menon V, Shukla U. Hand hygiene compliance among healthcare workers in an accredited tertiary care hospital. Indian J Critic Care Med. 2014;18(10):689.

33. Cruz JP, Bashtawi MA. Predictors of hand hygiene practice among Saudi nursing students: a cross-sectional self-reported study. J Infect Public Health. 2016;9(4):485-93.

34. Ellingson $\mathrm{K}$, Haas JP, Aiello AE, Kusek L, Maragakis LL, Olmsted RN, et al. Strategies to prevent healthcare-associated infections through hand hygiene. Infect Control Hosp Epidemiol. 2014;35(8):937-60.

35. Mathai E, Allegranzi B, Seto W, Chraïti M-N, Sax H, Larson E, et al. Educating healthcare workers to optimal hand hygiene practices: addressing the need. Infection. 2010;38(5):349-56.

36. Mu X, Xu Y, Yang T, Zhang J, Wang C, Liu W, et al. Improving hand hygiene compliance among healthcare workers: an intervention study in a Hospital in Guizhou Province, China. Braz J Infect Dis. 2016;20(5):413-8.

\section{Publisher's Note}

Springer Nature remains neutral with regard to jurisdictional claims in published maps and institutional affiliations. 